\title{
Eating for numbing: A community-based study of trauma exposure, emotion dysregulation, dissociation, body dissatisfaction and eating disorder symptoms
}

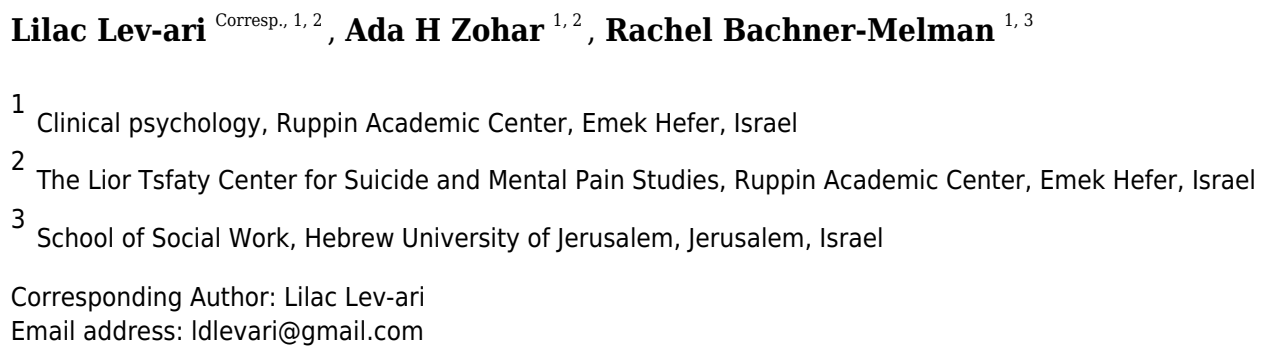

Objective: The current study tests the relationship between eating disorder (ED) symptoms and trauma exposure. The mechanisms via which trauma is related to ED symptoms have not been sufficiently examined. This study examines the complex role of dissociation and emotional dysregulation in the context of trauma, BMI, ED symptoms and body dissatisfaction (BD). We hypothesized that dissociation and emotional dysregulation would mediate the relationship between trauma exposure and ED symptoms / BD. We further hypothesized that BMI would play a moderating role in this association. Method: A community sample of 229 (16.2\% male) participants, with a mean age of $29.08 \pm 10.68$ reported online on traumatic events (Life Events Checklist), dissociation (Dissociative Experiences Scale - II), emotional dysregulation (Difficulties in Emotional Regulation Scale), ED symptoms (Eating Disorders Examination - Questionnaire) and BD (Figure Rating Scale). Results: Participants reported experiencing a mean of $2.87 \pm 2.27$ traumatic events, with a relatively high percentage $(\sim 86 \%)$ reporting at least one. The most commonly reported traumatic events were transportation accidents and physical assault. Although frequency of traumatic events did not directly predict ED symptoms, BMI, dissociation, emotional dysregulation and BD did. An SEM model showed that traumatic events predicted ED symptoms indirectly through dissociation, emotional dysregulation and BMI. Dissociation and emotional dysregulation predicted ED symptoms directly. BMI also moderated the association between traumatic events and both ED symptoms and BD. Conclusions: Therapists treating patients with high BMI or obesity should be aware of these relationships and investigate the possibility that trauma and/or PTSD may underlie the presenting disordered eating or eating disorder. 
A community-based study of trauma exposure, emotion dysregulation, dissociation, body 3 dissatisfaction and eating disorder symptoms

1 - Clinical Psychology Graduate Program, Ruppin Academic Center, Emek Hefer, Israel. 11

2 - The Lior Tsfaty Center for Suicide and Mental Pain Studies, Ruppin Academic Center, Emek 12 Hefer, Israel $\quad 13$

3 - School of Social Work, Hebrew University of Jerusalem, Jerusalem, Israel. 14

Corresponding author: Lilac Lev-Ari, PhD 19

Phone number: 972-52-8632434

Email address: 1dlevari@gmail.com

Conflict of interest statement: We have no conflict of interest

Acknowledgments: We have no acknowledgments to state

Funding information: We have no funding information to state 


\section{Abstract}

Objective: The current study tests the relationship between eating disorder (ED) symptoms and

trauma exposure. The mechanisms via which trauma is related to ED symptoms have not been

sufficiently examined. This study examines the complex role of dissociation and emotional

dysregulation in the context of trauma, BMI, ED symptoms and body dissatisfaction (BD). We

hypothesized that dissociation and emotional dysregulation would mediate the relationship

between trauma exposure and ED symptoms / BD. We further hypothesized that BMI would

play a moderating role in this association. Method: A community sample of 229 (16.2\% male)

participants, with a mean age of $29.08 \pm 10.68$ reported online on traumatic events (Life Events

Checklist), dissociation (Dissociative Experiences Scale - II), emotional dysregulation

(Difficulties in Emotional Regulation Scale), ED symptoms (Eating Disorders Examination -

Questionnaire) and BD (Figure Rating Scale). Results: Participants reported experiencing a

mean of $2.87 \pm 2.27$ traumatic events, with a relatively high percentage $(\sim 86 \%)$ reporting at least

one. The most commonly reported traumatic events were transportation accidents and physical

assault. Although frequency of traumatic events did not directly predict ED symptoms, BMI,

dissociation, emotional dysregulation and BD did. An SEM model showed that traumatic events

predicted ED symptoms indirectly through dissociation, emotional dysregulation and BMI.

Dissociation and emotional dysregulation predicted ED symptoms directly. BMI also moderated

Therapists treating patients with high BMI or obesity should be aware of these relationships and 
Keywords: Disordered Eating, Eating disorder symptoms, emotional dysregulation, body

dissatisfaction, dissociation, trauma

\section{Introduction}

Disordered eating (DE) behaviors and attitudes make up part of the eating disorder

continuum. Attitudes include obsessing about food and calories, becoming angry when hungry,

being unable to select what to eat, seeking food to compensate for psychological problems,

eating until feeling sick, and presenting unreal myths and beliefs about eating and weight

(American Dietetic Association, 2006). A more parsimonious definition is suggested by the work

of Hazzard et al. (2021), which is that any deviation from eating according to hunger and

satiation cues, i.e. intuitive eating, is disordered. In practice, disordered eating is measured by

assessing symptoms of eating disorders, behavioral, cognitive, and emotional (Alvarenga,

Scagliusi, \& Philippi, 2010), which do not reach the threshold of an eating disorder diagnosis.

The current study examines exposure to traumatic events, the absence of effective regulation of

negative emotion and the presence of dissociation as potential predictors of DE. These may serve

to numb the unprocessed distress arising from the experience of the trauma.

Whereas emotional dysregulation and dissociation may sometimes play a positive role in 
childhood abuse beyond PTSD and seem to impair cognition, mobility, daily functioning, self-

care, community involvement and interpersonal connection at least as much as PTSD symptoms

(Cloitre et al., 2005).

Whereas the role of trauma exposure has previously been implicated in eating disorder symptomatology, the mechanisms that may underlie this relationship remain uncertain. One such mechanism passes through dissociation, a of state of consciousness during which events and experiences usually integrated in consciousness are divided off and separated from one other.

This allows more than a single stream of consciousness, or multi-tasking. In her seminal book

“Trauma and Recovery", Herman (2015) explains the process of dissociation in the context of

trauma. After experiencing traumatic events, especially persistent trauma, individuals may adopt

dissociation as a routine way of dealing with everyday stressors. What begins as an adaptive

response can eventually impair the ability to focus on and respond appropriately to internal and

external circumstances.

Severe dissociative disorders are closely associated with exposure to trauma. Somer

(2004) found that the higher the intensity of the reported trauma, the stronger the dissociative

effects. He concluded that if the traumatic experiences are particularly prolonged and violent, the

the illusion of self-oneness and self-continuity, allowing the person to avoid experiencing

unbearable realities (Sommer, 2004). When parts of the self are disconnected and disowned, the

self develops further around the dissociative axis, fostering the creation of several narratives that

allow for the coexistence of the different parts of self. A dissociative inner organization may be

life-saving but also enables a pseudo-existence, a zone of half-life (Seligman, 2017). 
away from the horror of the traumatic experience, but on the other they disrupt processing and

recovery after the injury (Somer, 2004). If the event is experienced as shameful and secretive,

dissociation does not allow the trauma to be processed (Schwarzberg \& Somer, 2004).

Gruhn and Compas (2020) provide evidence that maltreatment early in life can trigger

105

processes that lead to emotion dysregulation. They point out that the emotional response patterns

of avoidance, suppression, and emotional coping can be helpful in surviving childhood

maltreatment. However, if generalized, they can become part of the diathesis for future

psychopathology. Alternations in neural development (Tupler \& De Bellis, 2006) and

impairments in biological stress response may occur (Tarullo \& Gunnar, 2006), and genetic

diathesis might render some individuals even more vulnerable than others to emotional

dysregulation (Halldorsdottir, 2017).

There is some support for a specific relationship between exposure to trauma and eating

pathology. In their review, Trottier and McDonald (2017) present evidence that exposure to

childhood trauma and severe adverse events are associated with bulimia nervosa $(\mathrm{BN})$, binge

eating disorder (BED) and anorexia nervosa (AN), particularly the binge-purge subtype (ANBP).

Furthermore, they present some evidence from longitudinal studies for a prospective association

between exposure to trauma as an adult and eating pathology. In adult women, sexual assault

was found to be related to binging and purging behaviors; the more violent the assault, the more

extreme was the eating pathology. Similar findings have been reported for disordered eating in

non-clinical samples. Hasselle et al. (2017) found that university students who reported more

childhood victimization also reported more disordered eating and higher levels of emotion 
PTSD symptoms and disordered eating, food addiction and overweight in elderly male veterans was mediated by the suppression of expressions of fear, hostility, guilt and sadness, underscoring a specific link between trauma, problems in emotional expression and disordered eating.

Palmisano et al. (2018) conducted a case control study of patients with obesity and no ED, patients with BED, and controls with average body mass index (BMI; $\mathrm{kg} / \mathrm{m} 2)$ They found that the participants with obesity reported higher levels of dissociation, and within the obese group, those with BED reported more childhood trauma than those without. Longo et al. (2019) found that in an ED treatment center, patients with ANBP reported more traumatic life events, especially sexual trauma, prior to the onset of the ED than patients with restrictive AN.

A relationship between exposure to trauma, psychological distress and high BMI has

been demonstrated in several contexts. Gu et al. (2014) conducted a national survey of law

psychological distress, likely related to exposure to traumatic events, were also heavier; when 
Moulton et al. (2015) and Pugh et al. (2018) found that dissociation mediated the

association between exposure to trauma during childhood and eating psychopathology in

adulthood. These studies showed an indirect relationship between exposure to trauma and in fact, be a form of emotion regulation.

The current study was conducted in Israel. Israel has a compulsory two/three-year mandatory military service with enlistment at 18 years of age, and the country experiences

relationships between exposure to trauma, emotion dysregulation, dissociation, disordered eating

1. Frequency of exposure to traumatic events would be significantly correlated with dissociation, emotional dysregulation, BD, ED symptoms and BMI.

2. BMI, frequency of exposure to traumatic events, dissociation, emotional dysregulation, and BD would predict ED symptoms (while controlling for demographic variables). 
3. Dissociation and emotional dysregulation would mediate the association between exposure to traumatic events and the other variables - BD, BMI and ED symptoms.

4. BMI would influence the association between frequency of exposure to traumatic events and $\mathrm{BD} / \mathrm{ED}$ symptoms.

\section{Methods}

\section{Participants}

A total of $233(40 ; 17.2 \%$ males $)$ participants between 20 and 72 years of age $(M=29.81$, $\mathrm{SD}=11.96$ ) registered online to participate in the study. Of these, 80 were community volunteers recruited via social networks and 153 were students enrolled in a BA level introductory were single, 50 (21.5\%) were married and nine (3.8\%) were divorced or had "other" status. They

\section{Measures}

\section{Traumatic Experiences}

Traumatic experiences were assessed using the Life Events Checklist (LEC; Gray et al., 
considered to have experienced this trauma. Frequency of traumatic experiences was

operationalized as the number of events participants indicated they had experienced. Gray et.al

(2004) assessed 108 undergraduate psychology students and found that the LEC had good

construct validity. We used the Hebrew translation of this questionnaire (Goren, 2003).

\section{Dissociation}

Dissociation was assessed using the Dissociative Experiences Scale - II (DES-II; Carlson

\& Putnam, 2001). The DES-II contains 28 items that describe different types of dissociative

experiences including amnesia (e.g. "Some people have the experience of driving or riding in a

car or bus or subway and suddenly realizing that they don't remember what has happened during

200

all or part of the trip"), depersonalization and de-realization (e.g. "Some people sometimes have

the experience of feeling as though they are standing next to themselves or watching themselves

do something and they actually see themselves as if they were looking at another person"), and

absorption ("Some people find that when they are watching television or a movie they become so

absorbed in the story that they are unaware of other events happening around them.”).

205

Respondents are asked what percent of the time they experience each kind of dissociative

experience on a categorical scale from $0 \%$ of the time i.e. never to $100 \%$ of the time i.e. always. 
Difficulties in emotional regulation were assessed using the Difficulties in Emotional

Regulation Scale (DERS; Gratz \& Roemer, 2004). The DERS contains 36 statements about

various aspects of emotional regulation, such as "When I'm upset, I lose control over my

behavior". Respondents rate their level of agreement with each statement on a scale from 1

(almost never) to 5 (almost always). The degree of difficulty in emotional regulation is

calculated by averaging the items in the questionnaire, with higher scores reflecting greater

difficulty regulating emotions. Reuveni et al. (2016) assessed 648 female university students and

220

found good internal reliability (.92). We used the Hebrew version of this questionnaire (Segal et.

al., 2016), which showed good internal reliability (Cronbach's alpha $=.90)$.

ED Symptoms were assessed using the Eating Disorders Examination - Questionnaire

(EDE-Q; Hilbert et al., 2007). The EDE-Q contains 28 items assessing the core symptoms of

overeating, dysregulation and compensation. The EDE-Q has four subscales, each containing

five to eight items: 1. Dietary Restraint (DR; "Have you been deliberately trying to limit the

amount of food you eat to influence your shape or weight [whether or not you have 
diagnostic purposes and are generally excluded from factor analysis. The cut-off score of four (in

subscales and in the global score) is suggested as being indicative of clinical EDs for both men

238

and women (Luce et al., 2008). In this study, disordered eating or "ED symptoms" refers to the

global EDE-Q score (average of all EDE-Q subscales). Zohar et. al. (2017) assessed 292

community volunteers and found sound psychometric properties for the Hebrew translation but

recommended combining WC and SC into one subscale (Zohar et. al., 2017). In the current study

the internal reliability was acceptable (Cronbach's alpha $>.78$ ).

\section{Body Dissatisfaction}

Body dissatisfaction was assessed using the Figure Rating Scale (FRS; Stunkard et. al.,

1983). The FRS presents silhouettes of increasing size, along a scale numbered from one through

nine. Respondents are asked to choose: (1) the figure that best represents their own body; (2) the

\section{Procedure}

The Institutional Internal Review Board approved the study and granted ethical approval 
weight, then completed the LEC, DES, EDE-Q and the DERS. After questionnaire completion,

contact details of the researchers were provided and participants were encouraged to reach out to

them with any questions, comments or difficulties.

Statistical Analysis

Missing data: Participants were prompted to respond to unanswered questions. All

participants answered the entire questionnaire with the exception of one participant for whom

one response was missing. The mean of this scale was assessed without the missing response.

Two participants did not answer the questions about education, three did not offer their age and

21 did not report how many children they had. Demographic data is reported without these missing responses.

Descriptive statistics were used to quantify frequency of trauma and Pearson correlations to quantify the associations between variables. Hierarchical Regression Analysis was used to predict ED symptoms using the independent variables. Structural equation model (SEM) was employed to assess the mediating effect of emotional dysregulation and dissociation on the association between trauma and both ED symptoms and BD. Mediation analyses were conducted to assess the effects of BMI on the association between trauma and BD / ED symptoms. Results

Hypothesis 1: Frequency of exposure to traumatic events would be significantly 
A high percentage of participants $(\sim 86 \%)$ reported having experienced at least one

traumatic event. Table 1 presents the percentage of participants who reported having experienced

Participants reported experiencing between 0-13 traumatic events (Mean=2.87,

$* * * * * *$ Figure 1 about here*********

One-sided Pearson correlations were calculated to assess the associations between

variables (see Table 2). The frequency of exposure to traumatic events was positively correlated

$* * * * * *$ Table 2 about here $* * * * * * * * *$

Hypothesis 2: BMI, frequency of exposure to traumatic events, dissociation, emotional 
To explore the effects of BMI on the various indices, we divided our sample into three

groups according to their BMI: High $(>25 ; n=20)$; intermediate (18.5-25; $n=193)$; and low

$(<18.5 ; \mathrm{n}=19)$ and assessed between-group differences on all variables (frequency of exposure to

traumatic events, dissociation, emotional dysregulation, body dissatisfaction and ED symptoms).

MANOVA analysis revealed overall significant differences between all three groups

$\left(\mathrm{F}_{(10,450)}=3.71, \mathrm{p}<.001\right)$, but only ED symptoms $\left(\mathrm{F}_{(2,228)}=10.81, \mathrm{p}<.001\right)$ and $\mathrm{BD}\left(\mathrm{F}_{(2,228)}=8.33\right.$,

$\mathrm{p}<.001$ ) differed significantly between the groups, echoing the findings of the Pearson

correlations.

To examine the hypothesis that BMI, frequency of exposure to traumatic events, dissociation, emotional dysregulation, and body dissatisfaction would predict ED symptoms (while controlling for demographic variables), a hierarchical regression analysis was conducted.

Demographic variables (age, sex and education) were introduced in the first step in order to control for them, making a case that they were not the reason for further associations between

variables. BMI was added in the second step as previous studies have found associations

between both BMI and trauma and between BMI and ED symptoms. Adding BMI in this step

allowed for assessing its added influence on explained variance and allowing to control for it

when assessing the association between trauma and ED symptoms. Frequency of exposure to

traumatic events and dissociation were added in the third step to assess their unique contribution

to the explained variance as were emotional dysregulation and BD in the last step. Results are

presented in Table 3 .

$* * * * * *$ Table 3 about here $* * * * * * * * *$

As can be seen from Table 3, BMI, dissociation, emotional dysregulation and BD were 
were associated with ED symptoms. The frequency of exposure to traumatic events did not

predict ED symptoms.

Hypothesis 3: Dissociation and emotional dysregulation would mediate the association

A central aim of this study was to build a comprehensive model depicting the

BMI and ED symptoms. A structural equation model (SEM) was designed, following the

dissatisfaction were hypothesized to be mediating variables, they were entered as such in the

analysis. Since BMI, BD and ED symptoms are known to be highly intercorrelated, they were

prediction of $\mathrm{BD}$ on emotional dysregulation, the path that was entered into the analysis in this

order. As a combined rule for the acceptance of our model, we chose the following

acknowledged values: normed fit index (NFI) > .90 (Bentler \& Bonett, 1980) and root mean

square error of approximation (RMSEA) <.08 (Browne \& Cudeck, 1993; see Figure 2). The Chi

Square goodness-of-fit index presented an excellent fit for the data, $\left.\chi_{(5)}^{2}=5.66, \mathrm{p}=.34\right)$; NFI $=.97$;

$\mathrm{CFI}=.99 ; \mathrm{RMSEA}=.02 ;$ standardized root means square residual $(\mathrm{RMR})=.03$.

$* * * * * *$ Figure 2 about here*********

The frequency of exposure to traumatic events positively predicted dissociation,

emotional dysregulation and BMI. BMI and BD were positively associated with one another, and 
predicted them indirectly through dissociation, emotional dysregulation and BMI. Dissociation

and emotional dysregulation directly predicted ED symptoms.

Hypothesis 4: BMI would influence the association between frequency of exposure to

traumatic events and BD / ED symptoms.

After noting the model of associations between frequency of traumatic events and ED

symptoms and BD, we further hypothesized that BMI would moderate the relationship between frequency of traumatic events and BD and ED symptoms.

Two moderation analyses were conducted. A hierarchical regression model was built to conduct a moderation analysis between frequency of traumatic events, BMI and BD. Frequency of traumatic events and BMI underwent centering procedures. In the first stage, frequency of traumatic events and BMI were entered as independent variables and BD (FRS) as the predictor.

The model was statistically significant $\left(\mathrm{F}_{(2,229)}=8.22, \mathrm{p}<.001\right)$ and explained $5.9 \%$ of the second model was statistically significant $\left(\mathrm{F}_{(3,228)}=20.77, \mathrm{p}<.001\right)$, adding an extra $15.57 \%$ to the

A follow-up analysis was conducted, aimed at estimating the conditional effects of the predictor variable (trauma frequency) on the outcome variable (BD) at low and high values of the moderator (BMI). The model for low BMI was not significant. For high BMI, the model was statistically significant $\left(\mathrm{F}_{(1,113)}=8.55, \mathrm{p}=.004\right)$, explaining $6.2 \%$ of the variance. Trauma 
A hierarchical regression model was then built to conduct a moderation analysis between frequency of traumatic events, BMI and ED symptoms. Frequency of traumatic events and BMI

A follow-up analysis aimed at estimating the conditional effects of the predictor variable significant.

As can be seen in Figure 3a and 3b: For people with low BMI, those with less traumatic events, also had the least ED symptoms. People with low BMI and many traumatic events had

\section{Discussion}


The main purpose of this study was to examine the association between the frequency of

traumatic events and both BD and disordered eating, as well as the influence of emotional

dysregulation, dissociation and BMI on this association.

Participants in this community study reported a wide range of traumatic experiences, with around half the sample reporting that they experienced up to two traumatic events in their

lifetime. The most common events reported were transport accidents, physical assault, and the

sudden death of a close person. Almost half the participants reported having had unwanted

399

sexual experiences, and 10\% a sexual assault. Over $40 \%$ reported having experienced trauma in

battle. This surprisingly large proportion can be explained by the fact that two or three years of

military service is compulsory for the participants, who were young Israeli adults, many of

whom had experienced combat. Beyond the idea of "national trauma" (Friedman-Peleg and Bilu.

2011) in a military sense, perpetual security concerns with repeated flare ups tend to make

civilian life more stressful and trauma exposure of all kinds more likely (Ben-Ya'acov et al.,

2005; Palmieri et al., 2008; Almogy, Kedar, \& Bala, 2016).

406

Contrary to our hypothesis, we found no direct link between the frequency of traumatic events and either ED symptoms or BD. We found, however, that emotional dysregulation and dissociation mediated the effect of traumatic events on ED symptoms and BD, and that BMI moderated the effect of traumatic events on ED symptoms and BD. Early studies of trauma and dissociation found that dissociation and past trauma characterized a high percentage of ED

patients (Tobin et. al., 1995; Vanderlinden et. al., 1993). Later studies focused specifically on

dissociative aspects of BN (Hallings-Pott et. al., 2005) and BED (La Mela et. al., 2010), positing

that dissociation may be a way of shifting awareness from negative feelings associated with the 
narrowing awareness and avoiding thoughts and feelings such as guilt and self-dislike (La Mela

et. al., 2010).

We found no direct associations between dissociation and emotional dysregulation with

BMI. Trauma, ED symptoms and BD were all associated directly with BMI, underscoring the

distressed, and emotional difficulties can lead either to high BMI (Czepczor-Bernat, 2019) or to

low BMI, as in restricted EDs (Farrington et. al., 2002) Such alternative patterns make linear

422

associations difficult to find, and path analysis can offer a clearer picture. Furthermore, when

participants were divided into high, intermediate and low BMI groups, significant differences

were observed only for disordered eating (ED symptoms) and BD, replicating the findings for linear correlations.

A link between trauma exposure and emotional dysregulation has been observed

consistently in research and clinical practice (Atchley and Bedford, 2020). Emotional

dysregulation has been shown to mediate the effect of childhood trauma on emotional eating

(Michopoulos et. al., 2015), so that the link between trauma and emotional eating seems partly

due to the strong associations between childhood trauma and emotional dysregulation. A

systematic review (Palmisano et. al., 2016) found that PTSD symptoms, dissociation, depression

and stress all mediate the effects of trauma on obesity and BED.

Arguably the most interesting finding of our study was that the frequency of exposure to

traumatic events was directly related to BMI, which in turn predicted ED symptoms and BD.

Since frequency of traumatic events was not directly associated with ED symptoms, it is our

belief that the model may reflect a complex route between trauma and ED symptoms that is not 
obesity, which found a positive association between traumatic events and obesity (Palmisano et al., 2016) and a mediating effect for PTSD and dissociation on the association between traumatic events and obesity. It should be noted, however, that whereas some studies have found that the rate of childhood trauma predicts obesity later in life (Jia et al., 2004), others have failed to find such an association (Goedecke et al., 2013). It is reasonable to assume that dissociation, feedback loop that accentuates both P TSD symptoms and ED behaviors.

Our moderation analysis attempts to further elucidate these connections. For people with low BMI, those who have experienced few or no traumatic events, ED symptoms were not evident as shown previously (Anderson et al., 2016). However, a different and more complex picture emerged for participants who had low BMI but had experienced many traumatic events. screening for trauma exposure.

This study has several limitations. First, we assessed the frequency of exposure to traumatic events but not their emotional impact or whether they were witnessed rather than directly experienced. A different pattern of results may have been observed had we included a 
about the types of traumatic events experienced is missing. Second, we did not assess post-

traumatic symptoms, which may be another trauma-related measure that impacts the study

variables. Third, this is a cross-sectional study and cannot lend itself to causational conclusions,

Fourth, we operationalized the experience of traumatic life events as their frequency, without

taking into consideration the type of traumatic events experienced. Although this approach has

been adopted in previous studies of obesity (Palmisano et al., 2018), it is less than ideal. Studies

that have examined the effects of specific types of trauma on EDs and BD have generally

468

focused on sexual trauma in childhood, which was not a major focus in this study. Lastly,

participants were young Israeli adults, most of whom had been enlisted in the army and grown

470

up in a country where trauma is all too frequent. The results may therefore not be generalizable

to other cultures.

This study offers a unique perspective on the influence of traumatic events on ED

symptoms and BD. Even though traumatic events may not influence ED symptoms and BD

directly, this study suggests that they may affect them indirectly via dissociation, emotion

\section{References}

Almogy, G., Kedar, A., \& Bala, M. (2016). When a vehicle becomes a weapon: intentional

vehicular assaults in Israel. Scandinavian Journal of Trauma, Resuscitation and Emergency

Medicine, 24(1), 149.

Alvarenga, M. D. S., Scagliusi, F. B., \& Philippi, S. T. (2010). Development and validity of the disordered eating attitude scale (DEAS). Perceptual and Motor Skills, 110(2), 379-395. 
American Dietetic Association. (2006) Position of the American Dietetic Association: nutrition

intervention in the treatment of anorexia nervosa, bulimia nervosa, and other eating

disorders. Journal of the American Dietetic Association, 106, 2073- 2082.

Amir, L. D., Aharonson-Daniel, L., Peleg, K., Waisman, Y., \& Israel Trauma Group. (2005). The severity of injury in children resulting from acts against civilian populations. Annals of Surgery, 241(4), 666.

Anderson, L. M., Reilly, E. E., Schaumberg, K., Dmochowski, S., \& Anderson, D. A. (2016).

Contributions of mindful eating, intuitive eating, and restraint to BMI, disordered eating, and meal consumption in college students. Eating and Weight Disorders, 21(1), 83-90.

Atchley, R., \& Bedford, C. (2020). Dissociative Symptoms in Posttraumatic Stress Disorder: A Systematic Review. Journal of Trauma \& Dissociation, 1-20.

Ben-Ya'acov, Y., Amir, M., Arzy, R., \& Kotler, M. (2005). Trauma in military and civilian 496 settings. Clinical Psychology \& Psychotherapy: An International Journal of Theory \& Practice, 12(1), 1-9.

Boyd, J. E., O'Connor, C., Protopopescu, A., Jetly, R., Lanius, R. A., \& McKinnon, M. C. (2020). The contributions of emotion regulation difficulties and dissociative symptoms to functional impairment among civilian inpatients with posttraumatic stress symptoms. Psychological Trauma: Theory, Research, Practice, and Policy. 
Cicchetti, D. (2016). Socioemotional, personality, and biological development: Illustrations from 506

a multilevel developmental psychopathology perspective on child maltreatment. Annual Review of Psychology, 67, 187-211.

Cloitre, M., Miranda, R., Stovall-McClough, K. C., \& Han, H. (2005). Beyond PTSD: Emotion regulation and interpersonal problems as predictors of functional impairment in survivors of childhood abuse. Behavior Therapy, 36(2), 119-124.

Czepczor-Bernat, K., Brytek-Matera, A., Gramaglia, C., \& Zeppegno, P. (2019). The moderating effects of mindful eating on the relationship between emotional functioning and eating styles in overweight and obese women. Eating and Weight Disorders-Studies on Anorexia, Bulimia and Obesity, 1-9.

Farrington, A., Waller, G., Neiderman, M., Sutton, V., Chopping, J., \& Lask, B. (2002).

Dissociation in adolescent girls with anorexia: Relationship to comorbid psychopathology. The Journal of Nervous and Mental Disease, 190(11), 746-751.

Fox, J. R., \& Power, M. J. (2009). Eating disorders and multi-level models of emotion: An integrated model. Clinical Psychology \& Psychotherapy: An International Journal of Theory \& Practice, 16(4), 240-267.

Friedman-Peleg, K., \& Bilu, Y. (2011). From PTSD to "national trauma": The case of the Israel Trauma Center for victims of terror and war. Transcultural Psychiatry, 48(4), 416-436.

Goedecke, J. H., Forbes, J., \& Stein, D. J. (2013). Differences in the association between

Goren, M. (2003). Stressful life events among depressed adolescents suffering from borderline 
Gratz, K. L., \& Roemer, L. (2004). Multidimensional assessment of emotion regulation and dysregulation: Development, factor structure, and initial validation of the difficulties in

54.

Gray, M. J., Litz, B. T., Hsu, J. L., \& Lombardo, T. W. (2004). Psychometric properties of the Life Events Checklist. Assessment, 11(4), 330-341.

Gerke, C. K., Mazzeo, S. E., \& Kliewer, W. (2006). The role of depression and dissociation in the

relationship between childhood trauma and bulimic symptoms among ethnically diverse female undergraduates. Child Abuse \& Neglect, 30(10), 1161-1172.

Gruhn, M. A., \& Compas, B. E. (2020). Effects of maltreatment on coping and emotion regulation in childhood and adolescence: a meta-analytic review. Child Abuse \& Neglect, 103, 104446.

Gu, J. K., Charles, L. E., Burchfiel, C. M., Andrew, M. E., Ma, C., Bang, K. M., \& Violanti, J. M. (2013). Associations between psychological distress and body mass index among law enforcement officers: The National Health Interview Survey 2004-2010. Safety and Health at Work, 4(1), 52-62.

Halldorsdottir, T., de Matos, A. P. S., Awaloff, Y., Arnarson, E. Ö., Craighead, W. E., \& Binder, 
bulimic eating disorders: An experimental study. International Journal of Eating Disorders, $38(1), 37-41$.

Hampson, S. E., Edmonds, G. W., Goldberg, L. R., Barckley, M., Klest, B., Dubanoski, J. P., \&

Hillier, T. A. (2016). Lifetime trauma, personality traits, and health: A pathway to midlife health status. Psychological Trauma: Theory, Research, Practice, and Policy, 8(4), 447.

Hasselle, A. J., Howell, K. H., Dormois, M., \& Miller-Graff, L. E. (2017). The influence of childhood polyvictimization on disordered eating symptoms in emerging adulthood. Child Abuse \& Neglect, 68, 55-64.

Hayes, A. F. (2017). Introduction to mediation, moderation, and conditional process analysis: A regression-based approach. Guilford Publications.

Hazzard, V. M., Telke, S. E., Simone, M., Anderson, L. M., Larson, N. I., \& Neumark-Sztainer, 561 D. (2021). Intuitive eating longitudinally predicts better psychological health and lower use of disordered eating behaviors: findings from EAT 2010-2018. Eating and Weight Disorders-Studies on Anorexia, Bulimia and Obesity, 26(1), 287-294.

Henschel, S., Doba, K., \& Nandrino, J. L. (2019). Emotion regulation processes and psychoform and somatoform dissociation in adolescents and young adults with cumulative maltreatment. Journal of Trauma \& Dissociation, 20(2), 197-211.

Herman, J. L. (2015). Trauma and recovery: The aftermath of violence--from domestic abuse to political terror. Hachette UK.

Hilbert, A., Tuschen-Caffier, B., Karwautz, A., Niederhofer, H., \& Munsch, S. (2007). 
Hoffman, Y. S., Diamond, G. M., \& Lipsitz, J. D. (2011). The challenge of estimating PTSD

prevalence in the context of ongoing trauma: The example of Israel during the Second

Intifada. Journal of Anxiety Disorders, 25(6), 788-793.

Jia, H., Li, J. Z., Leserman, J., Hu, Y., \& Drossman, D. A. (2004). Relationship of abuse history

575 and other risk factors with obesity among female gastrointestinal patients. Digestive Disease and Science, 49(5), 872-877. doi: 10.1023/B:DDAS.0000030102.19372.52

Kent, A., Waller, G., \& Dagnan, D. (1999). A greater role of emotional than physical or sexual abuse in predicting disordered eating attitudes: The role of mediating variables. International

Journal of Eating Disorders, 25(2), 159-167.

Kubzansky, L. D., Bordelois, P., Jun, H. J., Roberts, A. L., Cerda, M., Bluestone, N., \& Koenen,

K. C. (2014). The weight of traumatic stress: a prospective study of posttraumatic stress disorder symptoms and weight status in women. JAMA psychiatry, 71(1), 44-51.

La Mela, C., Maglietta, M., Castellini, G., Amoroso, L., \& Lucarelli, S. (2010). Dissociation in eating disorders: relationship between dissociative experiences and binge-eating episodes. Comprehensive Psychiatry, 51(4), 393-400.

Longo, P., Bertorello, A., Panero, M., Abbate-Daga, G., \& Marzola, E. (2019). Traumatic events and post-traumatic symptoms in anorexia nervosa. European Journal of Psychotraumatology, 10(1), 1682930.

Luce K. H., Crowther J. H., \& Pole M. (2008). Eating Disorder Examination Questionnaire (EDE-Q): Norms for undergraduate women. International Journal of Eating Disorders, 41, 273-276. 
Michopoulos, V., Powers, A., Moore, C., Villarreal, S., Ressler, K. J., \& Bradley, B. (2015). The

mediating role of emotion dysregulation and depression on the relationship between

childhood trauma exposure and emotional eating. Appetite, 91, 129-136.

Mitchell, K. S., Porter, B., Boyko, E. J., \& Field, A. E. (2016). Longitudinal associations among

posttraumatic stress disorder, disordered eating, and weight gain in military men and women. American Journal of Epidemiology, 184(1), 33-47.

Moulton, S. J., Newman, E., Power, K., Swanson, V., \& Day, K. (2015). Childhood trauma and eating psychopathology: A mediating role for dissociation and emotion dysregulation?. Child Abuse \& Neglect, 39, 167-174.

Palmieri, P. A., Canetti-Nisim, D., Galea, S., Johnson, R. J., \& Hobfoll, S. E. (2008). The psychological impact of the Israel-Hezbollah War on Jews and Arabs in Israel: The impact of risk and resilience factors. Social Science \& Medicine, 67(8), 1208-1216.

Palmisano, G. L., Innamorati, M., Sarracino, D., Bosco, A., Pergola, F., Scaltrito, D., ... \& Vanderlinden, J. (2018). Trauma and dissociation in obese patients with and without binge eating disorder: A case-control study. Cogent Psychology, 5(1), 1470483.

Palmisano, G. L., Innamorati, M., \& Vanderlinden, J. (2016). Life adverse experiences in relation with obesity and binge eating disorder: A systematic review. Journal of Behavioral Addictions, 5(1), 11-31.

Pugh, M., Waller, G., \& Esposito, M. (2018). Childhood trauma, dissociation, and the internal eating disorder 'voice'. Child Abuse \& Neglect, 86, 197-205.

Reuveni, I., Dan, R., Segman, R., Evron, R., Laufer, S., Goelman, G., ... \& Canetti, L. (2016). 
Sasson-Levy, O. (2007). Contradictory consequences of mandatory conscription: The case of

women

secretaries in the Israeli military. Gender \& Society, 21(4), 481-507.

Schrepf, A., Markon, K., \& Lutgendorf, S. K. (2014). From childhood trauma to elevated Creactive protein in adulthood: the role of anxiety and emotional eating. Psychosomatic Medicine, 76(5), 327.

Schwarzberg, S. \& Somer, A. (2004). Revealing the secret: Factors encouraging and delaying the discovery of the secret of the abuse among victims of childhood sexual abuse. In T.

Seligman and Z. Salomon (Eds.), The Secret and the Brake: Incest Issues. Tel Aviv, Israel: United Kibbutz and Adler Center, Tel Aviv University.

Segal, A., \& Golan, M. (2016). Differences in emotion regulation along the eating disorder spectrum: Cross sectional study in adolescents out patient care. Journal of Psychology \& Clinical Psychiatry, 6(1), 00314.

Seligman, T. (2017). In the absence of the witness there is no knowledge: The therapeutic tragedy of Ms. A. In O. Eshel and T. Seligman (Eds.), Was or was not? When shadows of childhood sexual abuse rise in treatment. Jerusalem, Israel: Carmel.

Somer, E. (2004). To be or not to be: Childhood traumas and disconnection disorders. In T.

Seligman and Z. Salomon (Eds.), The Secret and the Brake: Incest Issues. Tel Aviv, Israel: 53-65. 
Tarullo, A. R., \& Gunnar, M. R. (2006). Child maltreatment and the developing HPA axis. Hormones and Behavior, 50(4), 632-639.

Tobin, D. L., Molteni, A. L., \& Elin, M. R. (1995). Early trauma, dissociation, and late onset in the eating disorders. International Journal of Eating Disorders, 17(3), 305-308.

Trottier, K., \& MacDonald, D. E. (2017). Update on psychological trauma, other severe adverse experiences and eating disorders: state of the research and future research directions. Current Psychiatry Reports, 19(8), 45.

Tupler, L. A., \& De Bellis, M. D. (2006). Segmented hippocampal volume in children and adolescents with posttraumatic stress disorder. Biological Psychiatry, 59(6), 523-529.

Quick V. M., \& Byrd-Bredbenner, C. (2013). Eating Disorders Examination Questionnaire (EDE-Q): Norms for US college students. Eating and Weight Disorders, 18, 29-35.

Vallacher, R. R., \& Wegner, D. M. (1987). What do people think they're doing? Action identification and human behavior. Psychological Review, 94(1), 3.

Vanderlinden, J., Vandereycken, W., Van Dyck, R., \& Vertommen, H. (1993). Dissociative experiences and trauma in eating disorders. International Journal of Eating Disorders, 13(2), 187-193.

Zohar, A.H., Lev-Ari, L., \& Bachner-Melman, R. (2017). The EDE-Q in Hebrew: structural and convergent/divergent validity in a population sample. Israel Journal of Psychiatry, 54(3), 1521. 


\section{Table 1 (on next page)}

Percent of participants who reported having experienced traumatic events $(N=233)$ 
$\begin{array}{lr}\text { Table } 1 & 1\end{array}$

Percent of participants who reported having experienced traumatic events $(\mathrm{N}=233)$

\begin{tabular}{|c|c|}
\hline Traumatic event & Percent \\
\hline Transport accident & 67.63 \\
\hline Physical assault & 52.59 \\
\hline Sudden death of someone close to you & 50.32 \\
\hline Unwanted sexual experience & 45.63 \\
\hline Battle & 41.20 \\
\hline Life-threatening illness & 14.78 \\
\hline Fire & 14.78 \\
\hline Work accident & 14.78 \\
\hline Natural disaster & 13.66 \\
\hline Exposure to toxic material & 6.39 \\
\hline Sexual assault & 10.43 \\
\hline Assault with weapons & 7.87 \\
\hline Human agony & 5.91 \\
\hline Violent death of someone close to you & 1.30 \\
\hline You hurt or killed someone & 1.30 \\
\hline Captivity & 0.87 \\
\hline Other & 56.38 \\
\hline
\end{tabular}




\section{Table 2 (on next page)}

Pearson correlations between frequency of traumatic events and dissociation, emotional dysregulation, $B D, E D$ symptoms and $B M I(N=233)$ 
Table 2

Pearson correlations between frequency of traumatic events and dissociation, emotional

dysregulation, $B D$, ED symptoms and $B M I(N=233)$

\begin{tabular}{lllllll}
\hline & LEC & DES-II & DERS & FRS & EDE-Q & BMI \\
\hline Frequency of traumatic events & & $.17^{* *}$ & $.15^{* *}$ & -.08 & .09 & $.14^{* *}$
\end{tabular}

(LEC)

Dissociation (DES-II)

$.39 * * * \quad .10 \quad .28 * * * \quad-.02$

Emotional dysregulation (DERS)

$.22 * * * \quad .33 * * * \quad-.04$

Body dissatisfaction (FRS)

ED symptoms (EDE-Q)

Mean

Note: LEC $=$ Life Events Checklist; DES-II= Dissociative Experiences Scale - II (total score);

DERS $=$ Difficulties in Emotional Regulation Scale (total score); EDE-Q= Eating Disorders

$* \mathrm{p}<.05 ; * * \mathrm{p}<.01 ; * * * \mathrm{p}<.001$ 


\section{Table 3 (on next page)}

Hierarchical Regression Analysis predicting ED symptoms (global score) from BMI, frequency of traumatic events, dissociation, emotional dysregulation, and body dissatisfaction, while controlling for demographic variables $(N=233)$ 
Table 3

1

Hierarchical Regression Analysis predicting ED symptoms (global score) from BMI, frequency

2

of traumatic events, dissociation, emotional dysregulation, and body dissatisfaction, while

controlling for demographic variables $(N=233)$

\begin{tabular}{|c|c|c|c|c|c|c|}
\hline & Standardized $\beta$ & $\mathrm{T}$ & $\begin{array}{l}\text { Adj. } \\
R^{2}\end{array}$ & $\Delta \mathrm{R}^{2}$ & $F_{(d f)}$ & $\mathrm{p}$ \\
\hline Step 1 & & & .006 & & $1.49_{(3,223)}$ & NS \\
\hline Sex & .12 & 1.73 & & & & \\
\hline Education & -.08 & -.96 & & & & \\
\hline Age & .12 & 1.40 & & & & \\
\hline Step 2 & & & .12 & $.11 * * *$ & $8.38_{(4,222)} * * *$ & .00 \\
\hline Sex & .16 & $2.34^{*}$ & & & & .02 \\
\hline Education & -.05 & -.63 & & & & .53 \\
\hline Age & -.02 & -.22 & & & & .83 \\
\hline BMI & .36 & $5.34 * * *$ & & & & .00 \\
\hline Step 3 & & & .17 & $.06 * * *$ & $8.61_{(6,220)} * * *$ & .00 \\
\hline Sex & .15 & $2.26^{*}$ & & & & .03 \\
\hline Education & -.01 & -.07 & & & & .95 \\
\hline Age & -.04 & -.05 & & & & .96 \\
\hline BMI & .35 & $5.36 * * *$ & & & & .00 \\
\hline LEC & .01 & .21 & & & & .84 \\
\hline DES-II & .25 & $3.89 * * *$ & & & & .00 \\
\hline Step 4 & & & .23 & $.06 * * *$ & $10.61_{(7,219)} * * *$ & .00 \\
\hline Sex & .17 & $2.58 * *$ & & & & .01 \\
\hline Education & .03 & .43 & & & & .67 \\
\hline Age & .05 & .62 & & & & .54 \\
\hline BMI & .34 & $5.41 * * *$ & & & & .00 \\
\hline LEC & -.01 & -.20 & & & & .84 \\
\hline DES-II & .16 & $2.49 * *$ & & & & .01 \\
\hline DERS & .28 & $4.30 * * *$ & & & & .00 \\
\hline Step 5 & & & .31 & $.08 * * *$ & $13.78_{(8,218)} * * *$ & .00 \\
\hline Sex & .07 & 1.16 & & & & .25 \\
\hline Education & -.01 & -.10 & & & & .93 \\
\hline Age & .08 & 1.09 & & & & .28 \\
\hline BMI & .24 & $3.83 * * *$ & & & & .00 \\
\hline LEC & .01 & .23 & & & & .82 \\
\hline DES-II & .16 & $2.60 * *$ & & & & .01 \\
\hline DERS & .21 & $3.30 * * *$ & & & & .001 \\
\hline FRS & .32 & $5.21 * * *$ & & & & .00 \\
\hline
\end{tabular}


Note: Sex $(1=$ male; $2=$ female); BMI=body mass index; LEC=Life Events Checklist; DES-II= $\quad 6$

Dissociative Experiences Scale - II (total score); DERS= Difficulties in Emotional Regulation 7

Scale (total score); FRS= Figure Rating Scale. 8

$* \mathrm{p}<.05 ; * * \mathrm{p}<.01 ; * * * \mathrm{p}<.001$ 
Table 4 (on next page)

Frequency of traumatic events $(N=233)$ 
Figure 1

Frequency of traumatic events $(N=233)$

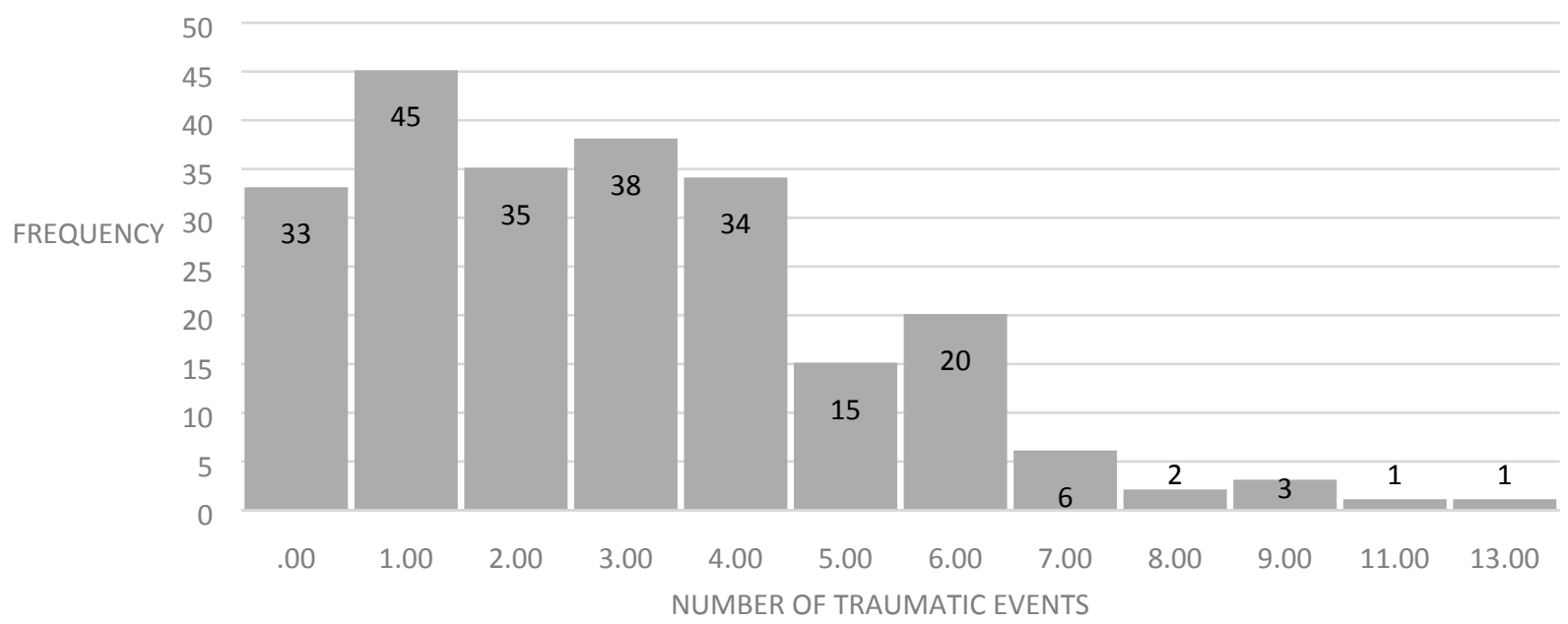




\section{Table 5 (on next page)}

SEM model depicting the relationships between frequency of traumatic events, dissociation, emotional dysregulation, $B D, B M I$ and $E D$ symptoms

Note: ${ }^{*} \mathrm{p}<.05 ;{ }^{* *} \mathrm{p}<.01 ;{ }^{* * *} \mathrm{p}<.001$. Frequency of traumatic events = LEC; Emotional dysregulation=DERS (total score); Dissociation=DES-II (total score); BMI=body mass index; Body dissatisfaction=FRS; ED symptoms=EDE-Q (global score) 
Figure 2

SEM model depicting the relationships between frequency of traumatic events, dissociation,

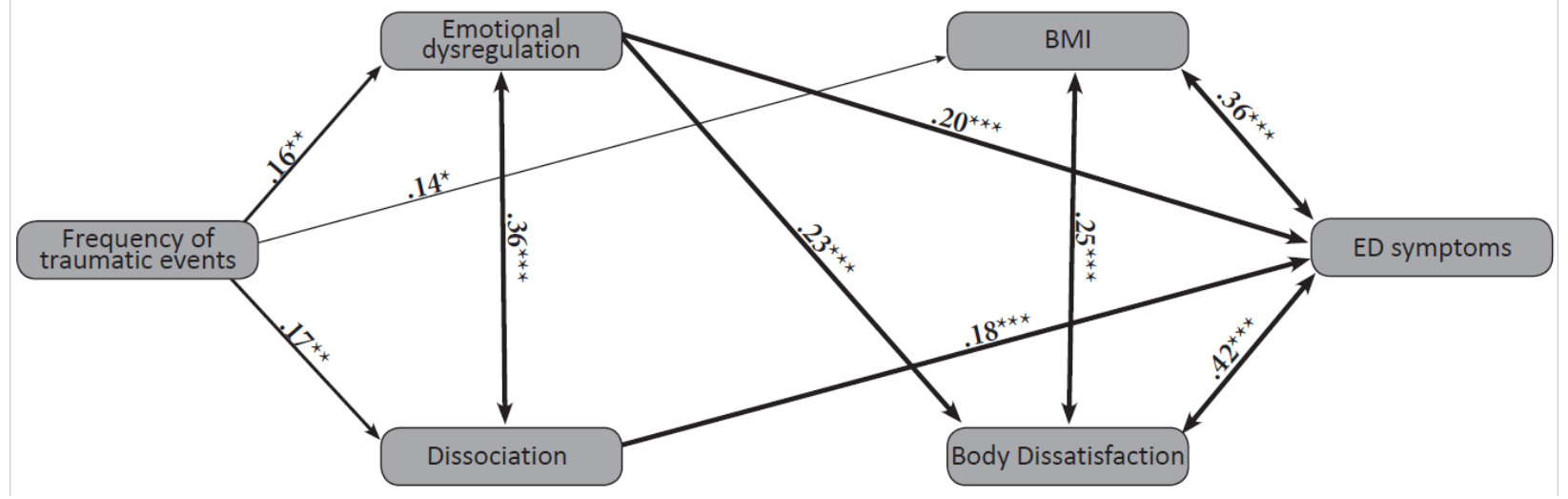

Note: ${ }^{*} \mathrm{p}<.05 ; * * \mathrm{p}<.01 ; * * * \mathrm{p}<.001$. Frequency of traumatic events $=$ LEC; Emotional

dysregulation=DERS (total score); Dissociation=DES-II (total score); BMI=body mass index;

Body dissatisfaction=FRS; ED symptoms=EDE-Q (global score) 


\section{Table 6(on next page)}

Moderation analysis: BMI moderates the association between frequency of traumatic events and $B D$ 


\section{Figure 3a}

1

Moderation analysis: BMI moderates the association between frequency of traumatic events and

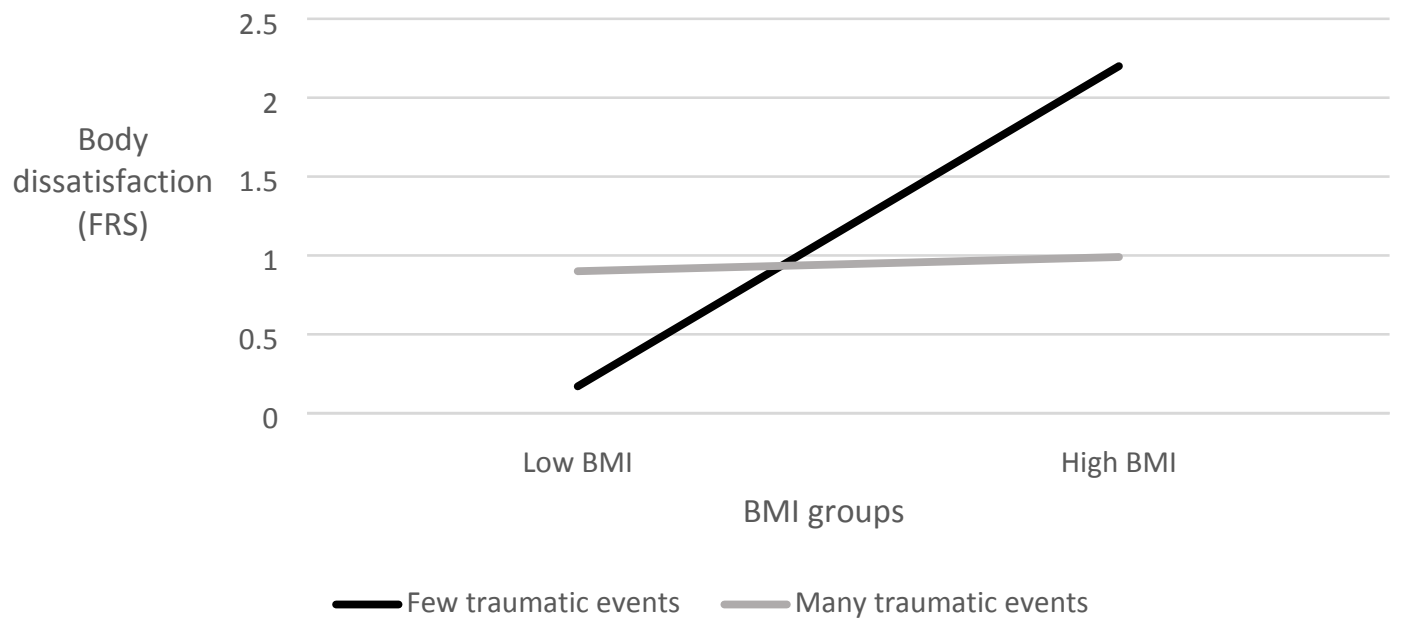




\section{Table 7 (on next page)}

Moderation analysis: $B M I$ moderates the association between frequency of traumatic events and ED symptoms (global score) 
$\begin{array}{lr}\text { Figure 3b } & 1\end{array}$

Moderation analysis: BMI moderates the association between frequency of traumatic events and

3

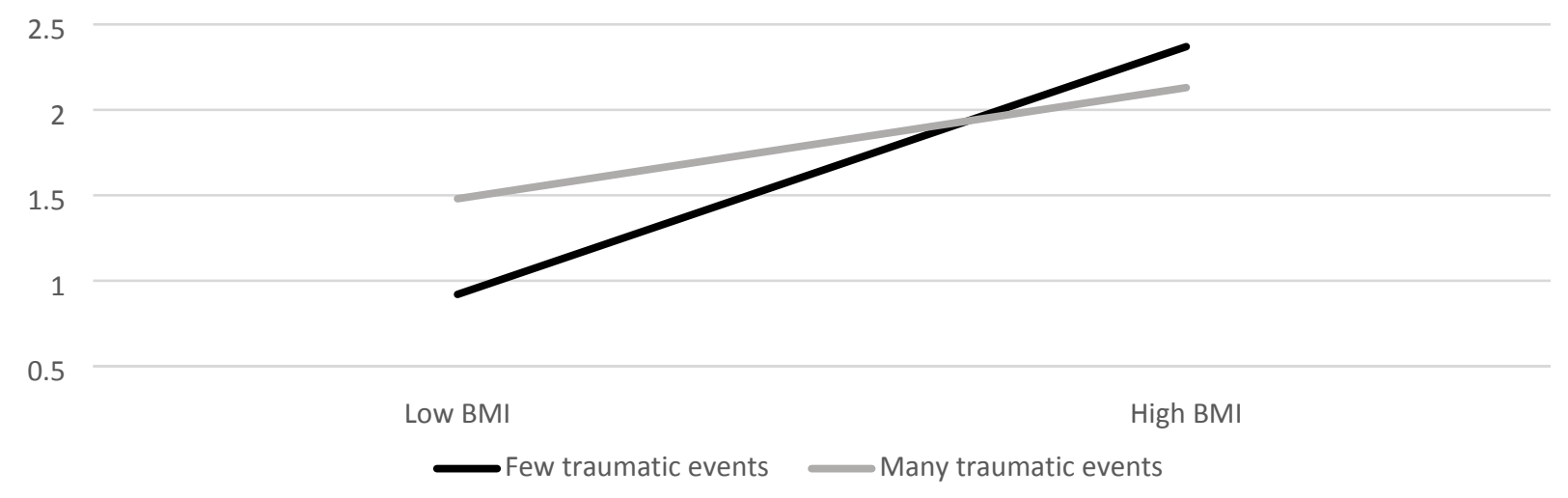

